\title{
An uncommon extrapulmonary sequestration located in the upper posterior mediastinum associated with the azygos lobe in a child
}

\author{
Yavuz Koksal, MD, ${ }^{a}$ Ekrem Unal, MD, ${ }^{\mathrm{b}}$ Olgun Kadir Aribas, MD, ${ }^{\mathrm{c}}$ and Bulent Oran, MD, ${ }^{\mathrm{b}}$ Konya, Turkey
}

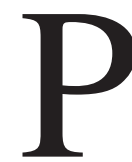

ulmonary sequestrations are rare congenital anomalies with an incidence of $0.15 \%$ to $1.7 \%$, and they are divided into intralobar and extralobar pulmonary sequestrations (ELPS). ${ }^{1}$ A nonfunctioning pulmonary tissue characterizes them without normal connection to the tracheobronchial tree and supplying blood from 1 or more abnormal systemic arteries. Most ELPS are located between the lower lobe of the lung and the diaphragm; however, they may occur anywhere within the thorax and partially or completely within the diaphragm. ${ }^{1,2}$

In approximately $25 \%$ of patients with ELPS, different congenital lung abnormalities, such as hypoplasia, congenital cystic adenomatoid malformation, congenital lobar emphysema, or bronchogenic cyst, associated with ELPS are known. ${ }^{2}$

In this report, we present a child with an ELPS located in the upper posterior mediastinum associated with the azygos lobe.

\section{Clinical Summary}

A 5-year-old boy was referred to our hospital with a murmur in the mesocardiac area. His medical history was unremarkable. Physical examination results were normal except for a functional systolic murmur $\left(1 /{ }^{\circ} 6\right)$. Blood biochemistry, complete blood count, and

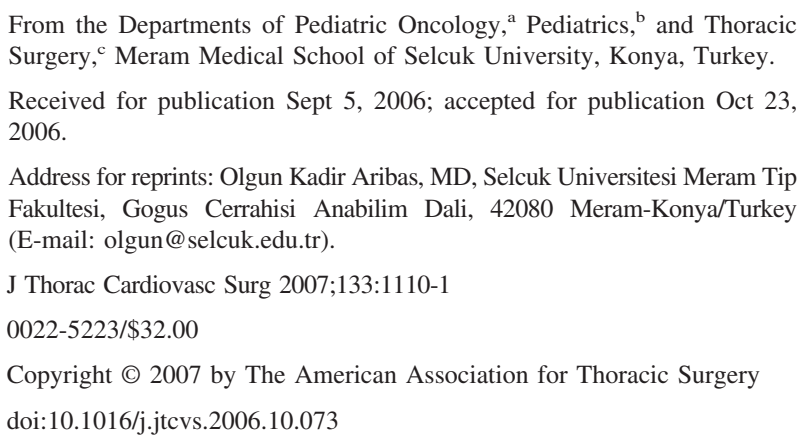

urinalysis results were normal. Chest radiography showed an upper posterior mediastinal mass (Figure 1, A). A diagnostic workup was done for malignancies, including urinary catecholamine metabolites, alpha-fetoprotein, and beta-human chorionic gonadotropin in serum, and all were within normal limits. Thorax computed tomography $(\mathrm{CT})$ revealed a heterogeneous mass, $6 \times 4 \times 3 \mathrm{~cm}$ in diameter, that had solid components and septations with contrast medium located peripherally (Figure 1,B). An accessory azygos lobe was explored during the thoracotomy. After the retraction of this lobe, the previously defined mass was seen to be ELPS tissue with blood supply from the ascending aorta and right brachiocephalic artery and draining to the superior vena cava through an accompanying vein. This sequestration tissue was lying in the posterior superior mediastinum, between the esophagus and the trachea, extending to the inferior of the right thyroid lobe. The lesion was not connected but adhered to the trachea by stub segmentary bronchia (Figure 2, A). Intraoperatively, dilatation of proximal esophagus secondary to the compression of the sequestration tissue was detected. After the dissection of bronchia and vessels, the sequestration lobe was totally excised (Figure 2, B). The postoperative follow-up period of 2 months was uneventful. No medication was given.

\section{Discussion}

ELPS is a rare congenital anomaly that commonly occurs on the left side; its location is between the lower lobe of the lung and the diaphragm in $63 \%$ to $77 \%$ of cases. ${ }^{1,2}$ Approximately $14 \%$ of ELPS may occur in the mediastinum. Only $6 \%$ of all ELPS are localized in the posterior mediastinum. ${ }^{2-4}$ Lupinski and colleagues ${ }^{3}$ reported a case of a 19-year-old asymptomatic man with a posterior mediastinal mass mimicking a neurogenic original mass on CT. However, they detected an ELPS in the posterior mediastinum by video-assisted thoracic surgery. A similar case was reported by York and colleagues. ${ }^{4}$ In their case, a hyperechoic midline mass was detected by fetal ultrasound at 21 weeks of

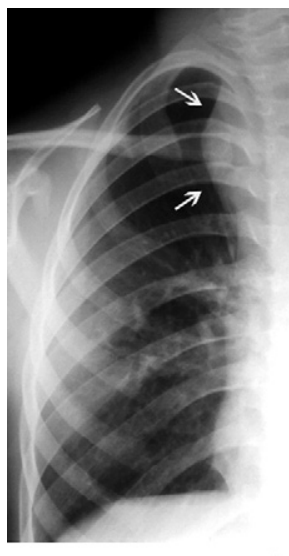

A

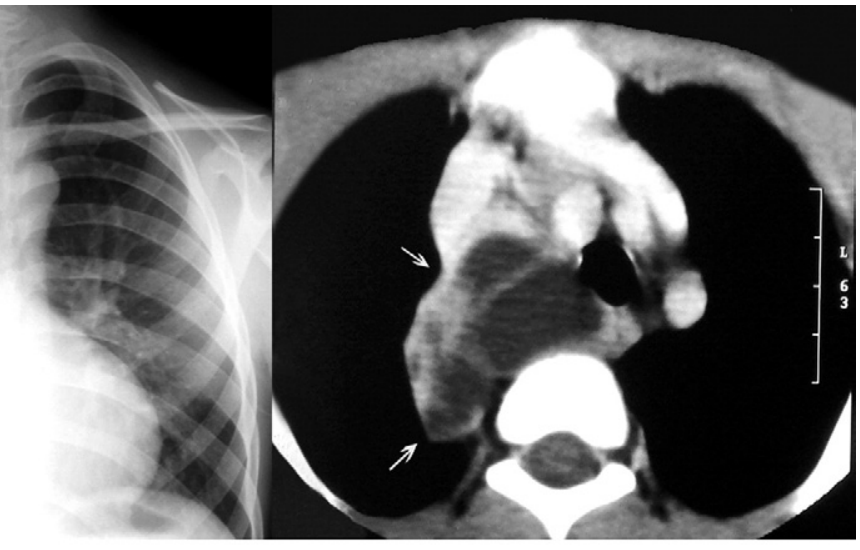

B
Figure 1. A, Chest x-ray film showing enlargement of the upper left mediastinum. B, Thoracic CT revealing a heterogeneous mass with solid components and septations with contrast medium located peripherally. 


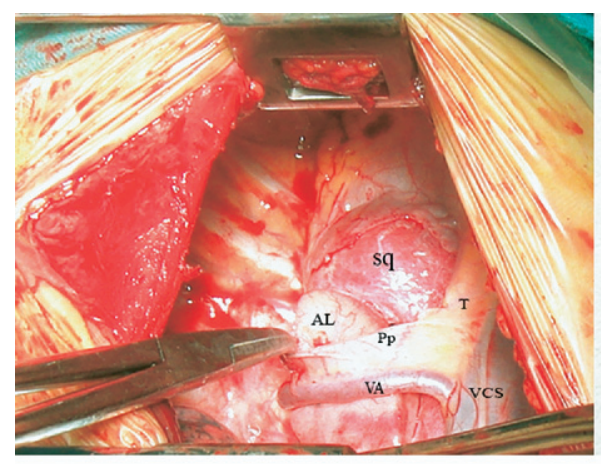

A

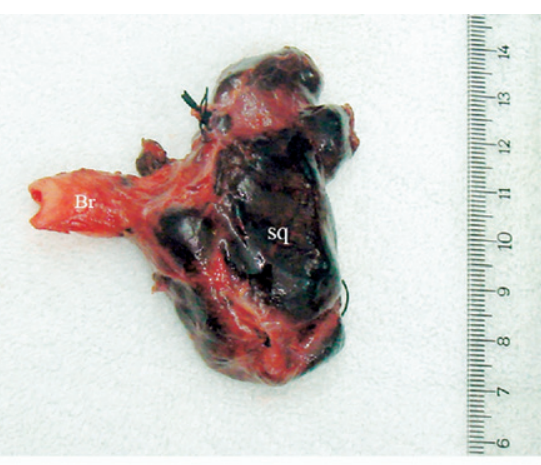

B
Figure 2. A, ELPS tissue associated with azygos lobe formed by an aberrant loop of the azygos vein beneath parietal pleura was lying in the posterior superior mediastinum, behind the trachea and vena cava superior. B, Macroscopic appearance of totally excised ELPS with the bronchia. ELPS, extralobar pulmonary sequestrations; $A L$, Azygos lobe; VA, azygos vein; $P$, parietal pleura; $T$, trachea; VCS, vena cava superior; sq, ELPS tissue; $B r$, bronchia. gestation. Magnetic resonance imaging performed at 29 weeks of gestation showed a posterior mediastinal mass. At 3 weeks of age, after diagnostic right thoracoscopy, the mass was totally excised. Histopathologic examination revealed pulmonary sequestration.

In our case, the posterior upper mediastinal mass was detected on chest radiography. Thoracic CT revealed a heterogeneous mass that had solid components and septations with contrast medium located peripherally.

The arterial supply to most ELPS (80\%) comes from the thoracic or abdominal aorta; the remains receive blood from the subclavian, brachiocephalic, splenic, gastric, intercostal, and pulmonary arteries. ${ }^{1,2}$ Venous drainage of ELPS is into the azygos or inferior vena cava and the pulmonary veins. ${ }^{2}$ In our case, ELPS tissue was receiving blood supply from the ascending aorta and right brachiocephalic artery, and draining to the superior vena cava by an accompanying vein.

ELPS has been reported to be associated with various congenital anomalies, of which congenital diaphragmatic hernia is the most frequent. ${ }^{1,2,5}$ The high occurrence of diaphragmatic hernia may suggest that a defect leading to ELPS occurs before the sixth week of gestation, before the diaphragm is fused. Other associated anomalies are congenital heart disease and pericardial defects, lung anomalies, skeletal malformations, foregut anomalies, gastrointes- tinal fistulous complications, and duplications. ${ }^{1,2,5}$ In our case, these congenital anomalies were not detected. However, an association between an ELPS and the azygos lobe was detected.

To our knowledge, this is the first reported case of an upper posterior mediastinal ELPS associated with the azygos lobe. Despite its rarity, an ELPS located in the upper posterior mediastinum can be easily mistaken for paraspinal or posterior mediastinal neoplastic disorders.

\section{References}

1. Wright C. Congenital malformations of the lung. Curr Diagn Pathol. 2006;12:191-201.

2. Corbett HJ, Humphrey GME. Pulmonary sequestration. Paediatr Respir Rev. 2004;5:59-68.

3. Lupinski RW, Agasthian T, Lim CH, Chua YL. Extralobar pulmonary sequestration simulates posterior neurogenic tumor. Ann Thorac Surg. 2004;77:2203-4.

4. York D, Swartz A, Johnson A, Fielding J, Phillips JD. Prenatal detection and evaluation of an extralobar pulmonary sequestration in the posterior mediastinum. Ultrasound Obstet Gynecol. 2006;27:214-6.

5. Weitzman JJ, Brennan LP. Bronchogastric fistula, pulmonary sequestration, malrotation of the intestine, and Meckel's diverticulum-a new association. J Pediatr Surg. 1998;33:1655-7. 